\title{
A FAST X-RAY DISK WIND IN THE TRANSIENT PULSAR IGR J17480-2446 IN TERZAN 5
}

\author{
Jon M. Miller ${ }^{1}$, Dipankar Maitra ${ }^{1}$, Edward M. CacketT $^{2}$, Sudip Bhattacharyya $^{3}$, And Tod E. Strohmayer ${ }^{4}$ \\ ${ }^{1}$ Department of Astronomy, University of Michigan, 500 Church Street, Ann Arbor, MI 48109-1042, USA; jonmm@umich.edu \\ ${ }^{2}$ Institute of Astronomy, University of Cambridge, Madingley Road, Cambridge, CB3 OHA, UK \\ ${ }^{3}$ Department of Astronomy and Astrophysics, Tata Institute of Fundamental Research, Mumbai 400005, India \\ ${ }^{4}$ Astrophysics Science Division, NASA Goddard Space Flight Center, Greenbelt, MD 20771, USA \\ Received 2011 January 11; accepted 2011 February 25; published 2011 March 17
}

\begin{abstract}
Accretion disk winds are revealed in Chandra gratings spectra of black holes. The winds are hot and highly ionized (typically composed of He-like and H-like charge states) and show modest blueshifts. Similar line spectra are sometimes seen in "dipping" low-mass X-ray binaries (LMXBs), which are likely viewed edge-on; however, that absorption is tied to structures in the outer disk, and blueshifts are not typically observed. Here, we report the detection of blueshifted He-like Fe Xxv $\left(3100 \pm 400 \mathrm{~km} \mathrm{~s}^{-1}\right)$ and H-like Fe XXVI $\left(1000 \pm 200 \mathrm{~km} \mathrm{~s}^{-1}\right)$ absorption lines in a Chandra/HETG spectrum of the transient pulsar and LMXB IGR J17480-2446 in Terzan 5. These features indicate a disk wind with at least superficial similarities to those observed in stellar-mass black holes. The wind does not vary strongly with numerous weak X-ray bursts or flares. A broad Fe K emission line is detected in the spectrum, and fits with different line models suggest that the inner accretion disk in this system may be truncated. If the stellar magnetic field truncates the disk, a field strength of $B=(0.7-4.0) \times 10^{9} \mathrm{G}$ is implied, which is in line with estimates based on X-ray timing techniques. We discuss our findings in the context of accretion flows onto neutron stars and stellar-mass black holes.
\end{abstract}

Key words: accretion, accretion disks - globular clusters: individual (Terzan 5) - pulsars: individual (IGR J17480-2446) - stars: neutron

Online-only material: color figures

\section{INTRODUCTION}

IGR J17480-2446 was discovered on 2010 October 10 in an INTEGRAL monitoring observation of the Galactic bulge (Bordas et al. 2010). The source was found to be consistent with the position of the globular cluster Terzan 5, which hosts the better-known transient and Type I X-ray burst source EXO 1745-248. An affiliation with EXO 1745-248 was apparently strengthened with the detection of Type I bursts (Chevenez et al. 2010; Strohmayer \& Markwardt 2010). Analysis of a prior Chandra image of Terzan 5, and additional Swift observations revealed, however, that the source was not EXO 1745-248, but rather a new transient source (Heinke et al. 2010; also see Pooley et al. 2010). The source was subsequently given the name IGR $\mathrm{J} 17480-2446$, in recognition of its discovery with INTEGRAL (Ferrigno et al. 2010).

Detailed timing analysis shows that IGR J17480-2446 is an $11 \mathrm{~Hz}$ pulsar (Strohmayer \& Markwardt 2010). It also appears that IGR J17480-2446 evolved from an "atoll" into a "Z" source, based on the path it traces in an X-ray color-color diagram and its rapid variability components (including the possible detection of a $\mathrm{kHz}$ quasi-periodic oscillation, or QPO, at $815 \mathrm{~Hz}$; see Altamirano et al. 2010). Previously, only XTE $\mathrm{J} 1701-462$ had been observed to evolve in this manner (Homan et al. 2010). Slower, $\mathrm{mHz}$ QPOs were also detected from IGR J17480-2446, caused by recurrent weak bursts (Linares et al. 2010).

Neutron stars in globular clusters are of particular importance because the distance to globular clusters is often known precisely. This can eliminate a major source of uncertainty when estimating a blackbody emission radius. The distance to Terzan 5 is likely $5.5 \mathrm{kpc}$ (Ortolani et al. 2007; also see Ransom 2007). Motivated by the rare opportunity to study a bursting neutron star in a globular cluster, we proposed a Chandra Director's Time observation of IGR J17480-2446.

\section{OBSERVATION AND DATA REDUCTION}

IGR J17480-2446 was observed with Chandra on 2010 October 24 (ObsID 13161, SeqNum 401282), starting at 10:41:43 (UTC), for a total exposure of $30.0 \mathrm{ks}$. The High Energy Transmission Gratings (HETG) were used to disperse the incident flux onto the Advanced CCD Imaging Spectrometer spectroscopic array (ACIS-S). To prevent photon pileup, the ACIS-S array was operated in continuous clocking or "GRADED_CC" mode, which reduced the nominal frame time from $3.2 \mathrm{~s}$ to $2.85 \mathrm{~ms}$. This mode is subject to calibration uncertainties, partially owing to charge transfer inefficiency. The zeroth-order flux is incident on the S3 chip, and frames from this chip can be lost from the telemetry stream if a source is very bright. We therefore used a gray window over the zeroth-order aimpoint; only 1 in 10 photons were telemetered within this region. For a longer discussion of this mode, please see, e.g., Miller et al. (2006b) and Miller et al. (2008).

Data reduction was accomplished using CIAO version 4.1. Time-averaged first-order High-Energy Grating (HEG) and Medium-Energy Grating (MEG) spectra were extracted from the Level-2 event file. Redistribution matrix files (rmfs) were generated using the tool "mkgrmf"; ancillary response files (arfs) were generated using "mkgarf." The first-order HEG spectra and responses were combined using the tool "add_grating_orders." The spectra were then grouped to require a minimum of 10 counts $\mathrm{bin}^{-1}$. Light curves of the dispersed photons were created using "dmexract." Spectra from periods with and without bursts (see below) were created by filtering the Level-2 event file using "dmcopy," specifying intervals derived using an independent code. 


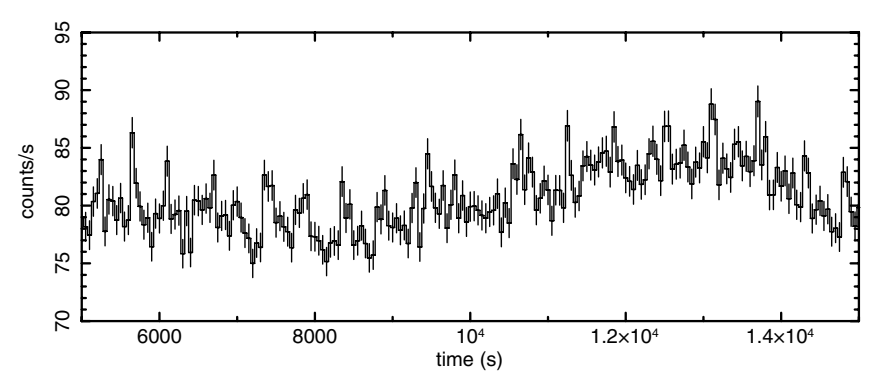

Figure 1. Typical segment of the light curve of the dispersed spectrum, with $50 \mathrm{~s}$ time bins. The rapid flaring seen is likely muted bursting activity and is the origin of the $\mathrm{mHz}$ QPOs detected in this source (see Linares et al. 2010).

All spectral analyses were conducted using XSPEC version 12.6.0. All errors quoted in this paper are $1 \sigma$ errors derived allowing all model parameters to vary simultaneously.

\section{ANALYSIS AND RESULTS}

\subsection{Bursts in the Light Curve}

The full $0.3-10.0 \mathrm{keV}$ light curve of IGR J17480-2446 was searched for bursts. Figure 1 shows a segment of the total light curve; several short, weak bursts are evident. Chakraborty \& Bhattacharyya (2011) suggest that the bursts seen in this transient are Type I bursts due to nuclear burning on the stellar surface, not Type II bursts due to an accretion disk process, as previously suggested by Galloway \& in't Zand (2010). Theoretical work shows that $\mathrm{mHz}$ QPOs can indeed be due to marginally stable nuclear burning (Heger et al. 2007). We employed the search algorithm used by Maitra \& Miller (2010) to flag bursts in the $0.3-10.0 \mathrm{keV}$ light curve. In total, 35 bursts were identified, generally confined within 100-200 s intervals. Time-selected spectra were made using the procedure described above.

\subsection{The Spectral Continuum}

Preliminary fits to the spectra revealed a rather high column density, approaching $10^{22} \mathrm{~cm}^{-2}$. For this reason, the MEG spectra are not very sensitive to narrow atomic lines. Moreover, the flux calibration of the MEG in the Fe $\mathrm{K}$ band differs from the better-calibrated HEG. As a result, our spectral analysis focused only on the summed first-order HEG spectrum. Below $1.3 \mathrm{keV}$ and above $9.0 \mathrm{keV}$, plausible continuum models leave residuals that are due to calibration uncertainties. More modest residuals are seen in the 1.7-3.0 keV band, likely owing to the complexity of modeling the effective area of CCDs in the Si K band, the Ir coating on the Chandra mirrors, and chip gaps that fall in this range. These features have little impact on the goodnessof-fit statistics. We therefore fit the HEG spectra across the $1.3-9.0 \mathrm{keV}$ band.

Although numerous bursts are evident in the source light curve, they are short-lived features that represent a small fluctuations on top of a high flux level. Fits to the burst-free spectrum of IGR J17480-2446 yield results that are consistent with fits to the time-averaged spectrum; the $0.5-10 \mathrm{keV}$ flux in the burst-free spectrum is only $5 \%$ lower than in the time-averaged spectrum. (The line properties measured during bursting phases, in the burst-free spectrum, and the time-averaged spectrum are also consistent.) Owing to this consistency, we focused on the time-averaged spectrum of IGR J17480-2446.

The continuum can be described well using a simple additive model, consisting of a blackbody component and a power law,

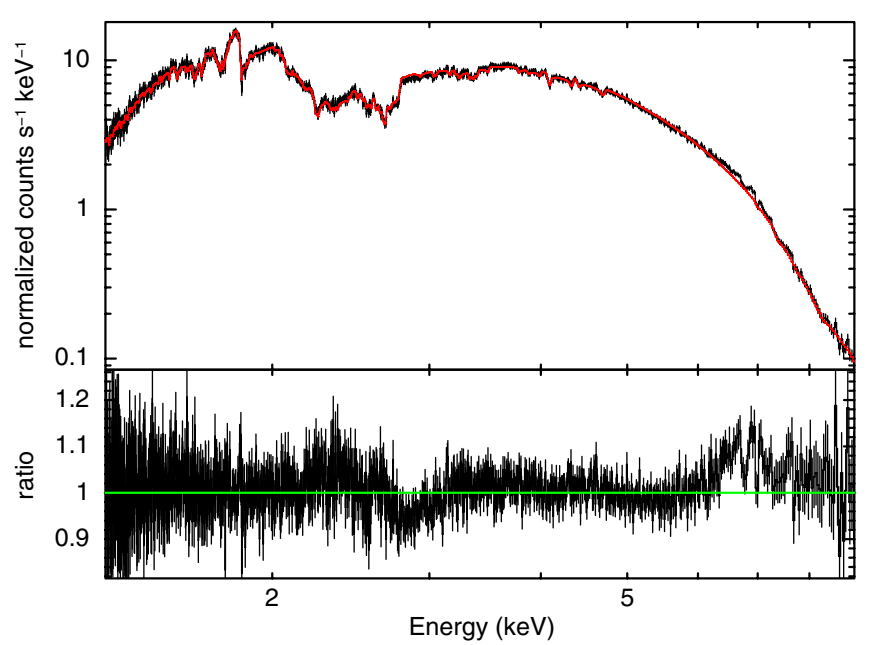

Figure 2. Time-averaged Chandra/HETGS spectrum of IGR J17480-2446, fitted with a simple blackbody plus power-law model. Note the broad Fe $\mathrm{K}$ emission line, and Fe XXV and Fe XXVI absorption lines in the data/model ratio. (A color version of this figure is available in the online journal.)

both modified by absorption along the line of sight. In XSPEC parlance, this model is "tbabs*(bbodyrad+powerlaw)," and it gives $\chi^{2} / v=3395.2 / 3255=1.04$ (where $v$ is the number of degrees of freedom). This model gives a column density of $N_{\mathrm{H}}=(1.17 \pm 0.04) \times 10^{22} \mathrm{~cm}^{-2}$, a blackbody temperature of $k T=1.18 \pm 0.01 \mathrm{keV}$, a blackbody normalization of $267 \pm 9$, a power-law index of $\Gamma=1.26 \pm 0.05$, and a powerlaw normalization of $0.46 \pm 0.05$. The normalization of the "bbodyrad" model gives a color radius of $9.0 \pm 0.1 \mathrm{~km}\left(K_{\mathrm{BB}}=\right.$ $R^{2} / D_{10}^{2}$, where $D_{10}$ is the distance in units of $\left.10 \mathrm{kpc}\right)$; this value is consistent with plausible stellar radii (e.g., Lattimer \& Prakash 2006). Extrapolating this simple model to the standard $0.5-10.0 \mathrm{keV}$ soft $\mathrm{X}$-ray band, it gives an unabsorbed flux of $1.01(5) \times 10^{-8} \mathrm{erg} \mathrm{cm}^{-2} \mathrm{~s}^{-1}$, which corresponds to a luminosity of $3.7 \pm 0.2 \times 10^{37} \mathrm{erg} \mathrm{s}^{-1}$. Figure 2 shows the combined firstorder HEG spectrum of IGR J17480-2446, fitted with this continuum.

\subsection{The Line Spectra}

The spectrum of IGR J17480-2446 shows clear residuals in the Fe K band (see Figures 2 and 3). A broad emission line, whether a simple Gaussian line or a relativistic line, gives an improvement to the overall fit that is significant at far more than the $8 \sigma$ level of confidence (the $F$-statistic is $7.8 \times 10^{-24}$ ). To characterize the absorption lines, we initially fit the broad line using a simple Gaussian function. The Gaussian model gives a centroid energy of $6.76(3) \mathrm{keV}$, which is broadly consistent with $\mathrm{Fe} \mathrm{XXV}$. The line strength is typical of neutron star low-mass $\mathrm{X}$-ray binaries (LMXBs), with an equivalent width of $112(9) \mathrm{eV}$. The width is measured to be $0.75(7) \mathrm{keV}$ (FWHM), or roughly $0.1 c$.

Fits to the likely $\mathrm{Fe}$ XXV absorption line with a simple Gaussian give a line centroid energy of $6.77(1) \mathrm{keV}$, indicating a blueshift of $3100 \pm 400 \mathrm{~km} \mathrm{~s}^{-1}$ (for a rest energy of $6.700 \mathrm{keV}$; Verner et al. 1996). The Gaussian fit nominally indicates that the line is resolved, with a width of $110 \pm 20 \mathrm{eV}$ or $4800 \pm 900 \mathrm{~km} \mathrm{~s}^{-1}$. An equivalent width of $12 \pm 3 \mathrm{eV}$ is measured. This line may be saturated; its properties may not be accurately measured using a Gaussian.

Fits to the likely Fe XXVI absorption line with a simple Gaussian give a centroid energy of $6.988(4) \mathrm{keV}$, indicating a 


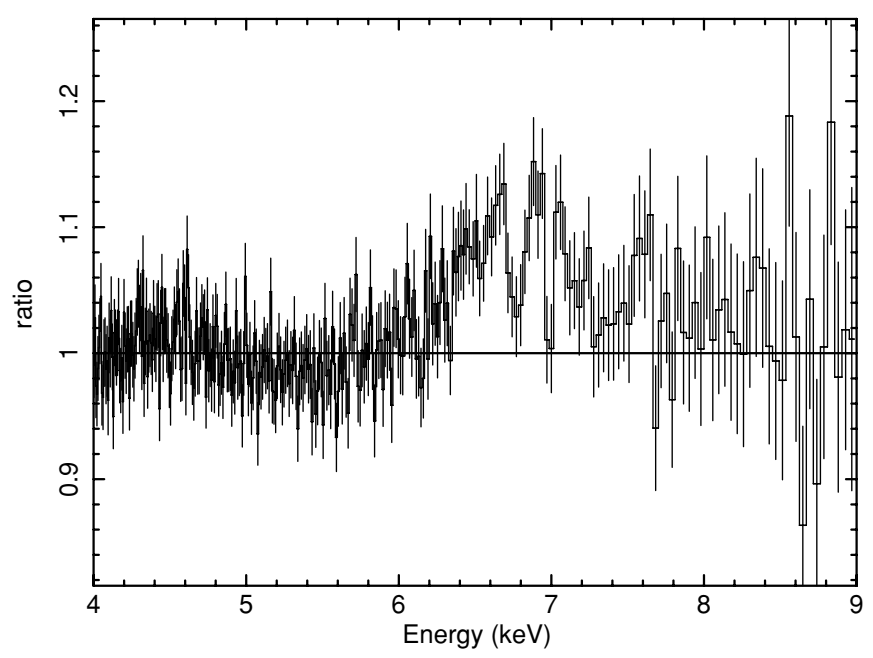

Figure 3. Fe K range in the data/model ratio shown in Figure 2. (The emission feature at $7.6 \mathrm{keV}$ is false and due to the addition of the first-order HEG spectra and responses.)

blueshift of $1000 \pm 200 \mathrm{~km} \mathrm{~s}^{-1}$ (for a rest energy of $6.966 \mathrm{keV}$; Verner et al. 1996). As Figures 3 and 4 indicate, this line is quite narrow, and it is not resolved. The $90 \%$ confidence upper limit on the width of this line is $13 \mathrm{eV}$ or $600 \mathrm{~km} \mathrm{~s}^{-1}$ (FWHM). An equivalent width of $6 \pm 2 \mathrm{eV}$ is measured for this line. This phenomenological continuum and complex of three Fe lines gives a very good fit: $\chi^{2} / v=3248.3 / 3248.0$. An apparent feature remaining at $6.1 \mathrm{keV}$ is not statistically significant. The inconsistency of the velocity shift in the He-like and $\mathrm{H}$-like lines could be real, but it may reflect possible saturation of the Fe XXV line. If it is real, the fact that the He-like line-which likely arises further from the ionizing flux than the $\mathrm{H}$-like line-has an higher velocity, may signal that the wind is being accelerated. Assuming that the observed absorption occurs on the linear part of the curve of growth, the equivalent width of the Fe XXV line implies a column of $N_{Z}=1.4(4) \times 10^{17} \mathrm{~cm}^{-2}$; that of the Fe XXVI line implies a column of $N_{Z}=1.3(4) \times 10^{17} \mathrm{~cm}^{-2}$.

Modeling the broad feature with a relativistic line function does not give a significantly better fit, compared to fits made with a simple Gaussian function. Detecting asymmetry in a broad line requires high sensitivity (see, e.g., Cackett et al. 2009b, 2010; also see Miller et al. 2010). Fits with the "diskline" model give $r_{\text {in }}=20(2) G M / c^{2}$, or $41(5) \mathrm{km}$ for a $1.4 M_{\odot}$ neutron star. This model also gives $i=18^{\circ} \pm 2^{\circ}, E=6.97_{-0.02} \mathrm{keV}$, and an equivalent width of $W=130 \pm 10 \mathrm{eV}$. The line emissivity index was fixed at $q=-3$, and the outer line emitting radius fixed at $1000 \mathrm{GM} / \mathrm{c}^{2}$.

We note that RXTE observed IGR J17480-2446 on 2010 October 24 (observation 95437-01-10-02). The Proportional Counter Array (PCA) spectra appear to also reveal a broad emission line (Chakraborty \& Bhattacharyya 2011). Simple fits to the PCU2 spectrum (including $0.6 \%$ systematic errors) in the $3-25 \mathrm{keV}$ band strongly require a line with a similar width $(0.9 \pm 0.2 \mathrm{keV}, \mathrm{FWHM})$ and strength (equivalent width $\mathrm{EW}=140 \pm 20 \mathrm{eV})$, but pegging at the lowest plausible energy for Fe K (6.40 keV).

The radius given by the relativistic line fit permits a constraint on the stellar magnetic field strength. Using Equation (1) in Cackett et al. (2009a), making the same assumptions regarding geometry and the accretion efficiency, adopting an unabsorbed $0.1-30.0 \mathrm{keV}$ flux of $1.9 \times 10^{-8} \mathrm{erg} \mathrm{cm}^{-2} \mathrm{~s}^{-1}$ as the bolometric flux, taking a distance of $5.5 \mathrm{kpc}$ (Ortolani et al. 2007),

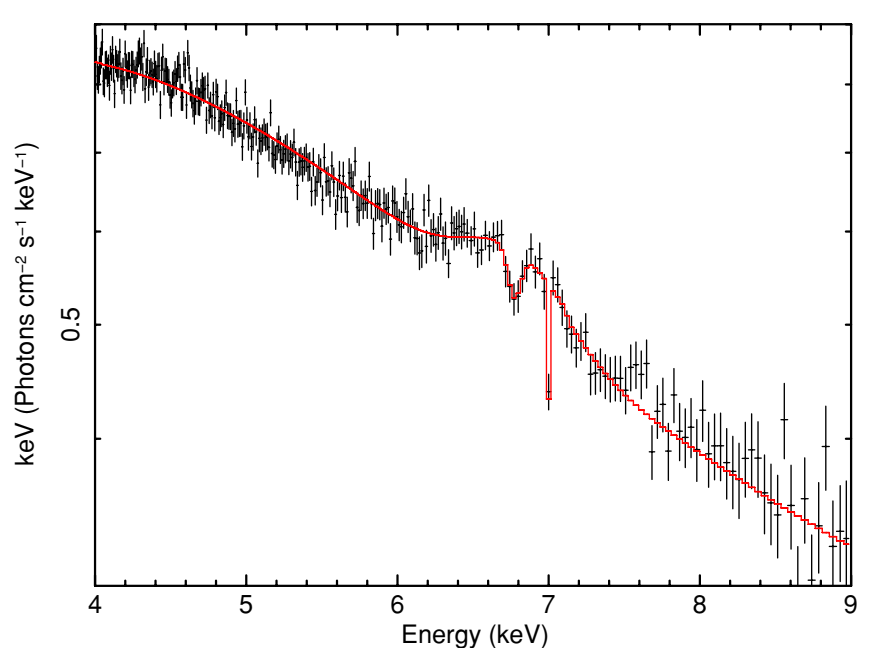

Figure 4. "Unfolded" or "fluxed" spectrum of IGR J17480-2446, based on phenomenological modeling to the spectrum in the Fe K band. A broad Gaussian emission line and two narrow Gaussian absorption lines provide a good fit to the atomic features.

(A color version of this figure is available in the online journal.)

and assuming a mass of $1.4 M_{\odot}$, we obtain $\mu=4.5(8) \times$ $10^{26} \mathrm{G} \mathrm{cm}^{-3}$. For a neutron star with a radius of $10 \mathrm{~km}$, this gives a magnetic field strength of $B=9(2) \times 10^{8} \mathrm{G}$ at the poles. If a value of 0.5 (Long et al. 2005) is assumed for the conversion factor from spherical to disk accretion when balancing magnetic and ram pressures, the magnetic field at the poles would be $B=3(1) \times 10^{9} \mathrm{G}$. Both values are consistent with estimates based on X-ray timing (Papitto et al. 2011).

\subsection{Photoionization Models}

In order to better model the absorption found in IGR J17480-2246, we constructed a grid of multiplicative models using XSTAR version 2.2 (Kallman \& Bautista 2001). The blackbody plus power-law model detailed above was extrapolated to the $0.1-30 \mathrm{keV}$ band, and supplied to XSTAR as the source of ionizing flux. Solar abundances were assumed. The outflow must be equatorial, owing to the lack of narrow emission lines in the spectra. We therefore assumed a global covering factor of 0.3 . Last, the grids considered here assumed a number density of $n=10^{12} \mathrm{~cm}^{-3}$, consistent with values used to characterize other winds in X-ray binaries.

Direct fits to the combined first-order HEG spectrum with the multiplicative table model strongly suggest that the Fe XXv and Fe XXVI lines observed cannot arise in exactly the same gas. Any single absorption zone that fits the Fe XXV line well also predicts a strong Fe XXVI line at a commensurate blueshift, which exceeds the shift measured from the observed Fe XXVI line. Similarly, any absorption zone that fits the observed Fe XXVI line well, underpredicts the Fe XXV line and fails to match its large observed blueshift.

A plausible fit to the spectrum $\left(\chi^{2} / v=3306.1 / 3246\right)$ can be obtained using two absorption zones and a relativistic diskline model for the broad emission line (see Figure 5). The Fe XXv line is well described by an absorber with $N_{\mathrm{H}}=3 \times 10^{22} \mathrm{~cm}^{-2}$, $\log (\xi)=3.0$, at a blueshift of $3100 \mathrm{~km} \mathrm{~s}^{-1}$. The Fe XXVI line is well described by a slightly more modest absorber: $N_{\mathrm{H}}=2 \times 10^{22} \mathrm{~cm}^{-2}, \log (\xi)=4.3$, at a blueshift of $500 \mathrm{~km} \mathrm{~s}^{-1}$. The higher velocity absorption zone includes a strong Fe XXVI line that is not evident. This may be explained if the blue 


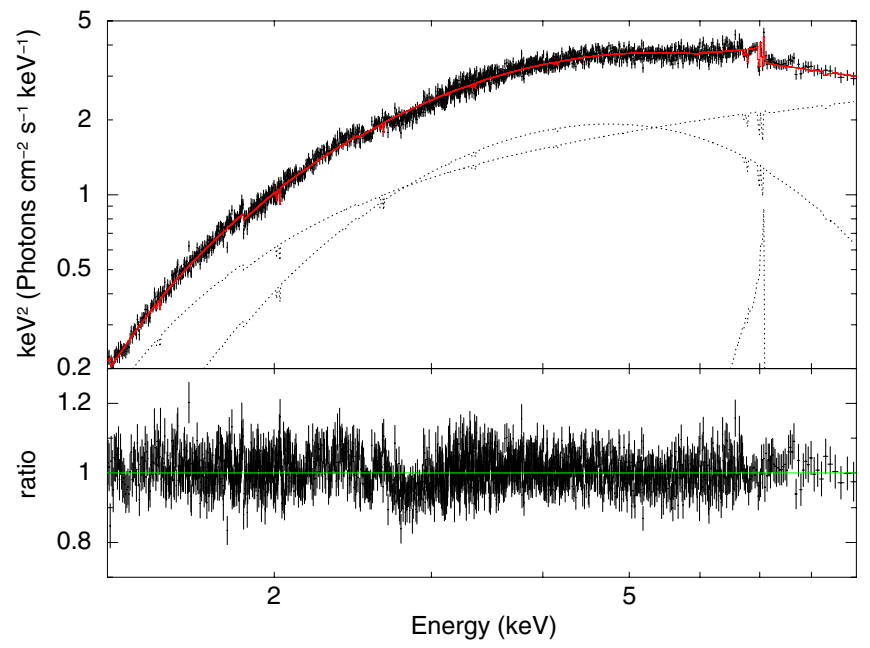

Figure 5. Unfolded spectrum of IGR J17480-2446 and the resulting data/ model ratio. The model used consists of a simple continuum, a relativistic disk line, and a two-zone ionized absorber generated using XSTAR. The model does an excellent job of fitting the complex spectrum observed in the Fe $\mathrm{K}$ band.

(A color version of this figure is available in the online journal.)

wing of a relativistic emission line (like that described above) coincides with the Fe XXVI absorption. The model described here is not unique (for this reason, errors are not given), and slightly different relativistic emission line profiles and absorption zones can be combined to give similar outcomes.

Assuming that the absorbing gas is a filled volume of uniform density, the ionization parameter can be used to derive a radius within which the wind must be launched $(r \simeq L / N \xi$, where $L$ is the source luminosity, $N$ is the column density, and $\xi$ is the ionization parameter). Even the more highly ionized absorber is relatively far away from the central engine: $r \simeq 3 \times 10^{5} \mathrm{~km}$ or $r \simeq 1.5 \times 10^{5} R_{\text {Schw. }}$ (assuming a neutron star mass of $\left.1.4 M_{\odot}\right)$. This is within the binary system of IGR $17480-2446$ : $a \sin (i)=2.498(5) \mathrm{s}$ or $7.5 \times 10^{5} \mathrm{~km}$ (Papitto et al. 2011), and consistent with the outer disk.

The mechanical luminosity in a wind is given by $L_{W}=$ $0.5 \dot{m} v^{2}$. In practice, it is necessary to rewrite this equation in terms of the ionization parameter and a geometric factor: $L_{W}=0.5 m_{p} \Omega v^{3}(L / \xi)$, where $m_{p}$ is the mass of the proton, $\Omega$ is the fraction of $4 \pi$ covered by the wind $(\Omega=0.3$ is assumed in our XSTAR grids), $v$ is the wind velocity, $L$ is the ionizing luminosity, and $\xi$ is the ionization parameter. The faster, less ionized absorption zone gives a mechanical luminosity of $L_{W} \simeq 2.8 \times 10^{35} \mathrm{erg} \mathrm{s}^{-1}$, or $L_{W} / L_{\text {accr. }} \simeq 8 \times 10^{-3}$.

\section{DISCUSSION AND CONCLUSIONS}

We have analyzed a Chandra/HETG observation of the transient X-ray pulsar and LMXB IGR J17480-2446 in Terzan 5. The Fe $\mathrm{K}$ band can be decomposed into a broad emission line consistent with He-like emission excited at inner edge of a radially truncated accretion disk, and blueshifted He-like and $\mathrm{H}$-like $\mathrm{Fe}$ absorption lines that likely arise in an X-ray disk wind. The implications of a truncated inner accretion disk in IGR J17480-2446 and a disk wind are of particular interest, and they are explored in this section.

At $11 \mathrm{~Hz}$, IGR J17480-2446 is a much slower pulsar than, e.g., SAX J1808.4-3658, which has a frequency of $401 \mathrm{~Hz}$ (Wijnands \& van der Klis 1998), and in which relativistic line spectroscopy and X-ray timing both imply a smaller inner disk radius and smaller stellar magnetic field (Cackett et al. 2009a;
Papitto et al. 2009; Hartman et al. 2008). In SAX J1808.4-3658, then, it is not surprising that the source shows $\mathrm{kHz}$ QPOs, which are often associated with orbits in the inner disk, and connections between the disk and the star.

At a flux level similar to that measured in this Chandra observation (after accounting for a distance disparity), Altamirano et al. (2010) reported the detection of QPOs at $48 \mathrm{~Hz}, 173 \mathrm{~Hz}$, and $815 \mathrm{~Hz}$. If the fastest QPO signals a Keplerian orbital frequency, a radius of approximately $9 G M / c^{2}$ is implied. If the inner disk was still truncated at 20(2) $G M / c^{2}$, then the $\mathrm{kHz}$ QPOs would have to be produced interior to the disk truncation radius. Fourier-resolved spectroscopy has shown that the variable part of such spectra may originates in the boundary layer (Revnivtsev \& Gilfanov 2006); thus, it is possible that $\mathrm{kHz}$ QPOs originate in the boundary layer. A more likely explanation is that the disk simply extended closer to the neutron star when $\mathrm{kHz}$ QPOs were detected.

The disk wind found in IGR J17480-2446 may be the clearest detection of such a flow in a neutron star system. Iron absorption lines from highly ionized gas are clearly detected in a number of "dipping" neutron star systems, but this absorption is not typically blueshifted, and likely occurs in the outer accretion disk of these nearly edge-on sources (see, e.g., Diaz Trigo et al. 2006). An ionized outflow is clearly detected in the persistent spectrum of GX 13+1 (Ueda et al. 2004); however, this source shows frequent dips, and this casts some doubt on the nature of the absorption. Similarly, an ionized outflow is detected in Circinus X-1 (Brandt \& Schulz 2000), but it is difficult to rule out a massive companion wind as the source of the outflow. An ionized X-ray wind was detected in a Chandra spectrum of the pulsar 1A 0535+262 (Reynolds \& Miller 2010). The flow is likely a disk wind, but it is difficult to entirely rule out absorption in the wind of the massive B companion star in 1A $0535+262$.

It is interesting to explore why disk winds are not regularly detected in the relatively large sample of persistent " $Z$ " and "atoll" neutron stars. A recent survey of Chandra/HETG spectra does not find evidence for ionized outflows (Cackett et al. 2009 b); in some cases, the sensitivity of the spectra may have simply been insufficient. However, even in deep observations of Cygnus X-2, blueshifted absorption is not reported in the non-dip spectra (Schulz et al. 2009).

An intriguing possibility is that the outflow observed in IGR J17480-2446 may be a different kind of disk wind than those observed in stellar-mass black holes. The winds seen in stellar-mass black holes originate close to the black hole (e.g., within $1000 \mathrm{GM} / \mathrm{c}^{2}$; see Miller et al. 2006a, 2006b, 2008; Kubota et al. 2007; Neilsen \& Lee 2009) and can carry away a good fraction of the accreting gas. Thus, the winds in stellarmass black holes may be at least partially driven by magnetic pressure (see Miller et al. 2008). In contrast, X-ray absorption in the disk wind found in IGR J17480-2446 originates two orders of magnitude further from the central engine (measured in units of $G M / c^{2}$ ). The high ionization parameter of the wind in IGR J17480-2446 makes it unlikely that radiation pressure can drive the wind (e.g., Proga 2000), but Compton heating of the outer disk could plausibly drive such a wind (e.g., Begelman et al. 1983).

We thank Harvey Tananbaum, Belinda Wilkes, and Andrea Prestwich for executing this observation. We acknowledge David Pooley and Jeroen Homan for helping to coordinate different observations of IGR J17480-2446. We thank Tim Kallman and Cole Miller for helpful discussions. Finally, we thank 
the anonymous referee for a helpful review. J.M.M. acknowledges support from the Chandra Guest Observer program.

\section{REFERENCES}

Altamirano, D., et al. 2010, ATel, 2952

Begelman, M. C., McKee, C. F., \& Shields, G. A. 1983, ApJ, 271, 70

Bordas, P., et al. 2010, ATel, 2919, 1

Brandt, W. N., \& Schulz, N. S. 2000, ApJ, 544, L123

Cackett, E. M., Altamirano, D., Patruno, A., Miller, J. M., Reynolds, M., Linares, M., \& Wijnands, R. 2009a, ApJ, 624, L21

Cackett, E. M., et al. 2009b, ApJ, 690, 1847

Cackett, E. M., et al. 2010, ApJ, 720, 205

Chakraborty, M., \& Bhattacharyya, S. 2011, ApJ, 730, L23

Chevenez, J., et al. 2010, ATel, 2924, 1

Diaz Trigo, M., Parmar, A. N., Boirin, L., Mendez, M., \& Kaastra, J. S. 2006, A\&A, 445, 179

Ferrigno, C., et al. 2010, ATel, 2940, 1

Galloway, D., \& in't Zand, J. J. M. 2010, ATel, 3000, 1

Hartman, J., et al. 2008, ApJ, 675, 1468

Heger, A., Cumming, A., \& Woosley, S. E. 2007, ApJ, 665, 1311

Heinke, C. O., Linares, M., Gladstone, J., Homan, J., Altamirano, D., \& Pooley, D. 2010, ATel, 2933, 1

Homan, J., et al. 2010, ApJ, 719, 201

Kallman, T., \& Bautista, M. 2001, ApJS, 133, 221

Kubota, A., et al. 2007, PASJ, 59S, 185

Lattimer, J. M., \& Prakash, M. 2006, Nucl. Phys. A, 777, 479

Linares, M., et al. 2010, ATel, 2958, 1

Long, M., Romanova, M. M., \& Lovelace, R. V. E. 2005, ApJ, 634, 1214
Maitra, D., \& Miller, J. M. 2010, ApJ, 718, 551

Miller, J. M., Raymond, J., Fabian, A., Steeghs, D., Homan, J., Reynolds, C., van der Klis, M., \& Wijnands, R. 2006a, Nature, 441, 953

Miller, J. M., Raymond, J., Reynolds, C. S., Fabian, A. C., Kallman, T. R., \& Homan, J. 2008, ApJ, 680, 1359

Miller, J. M., et al. 2006b, ApJ, 646, 394

Miller, J. M., et al. 2010, ApJ, 724, 1441

Neilsen, J., \& Lee, J. C. 2009, Nature, 458, 481

Ortolani, S., Barbuy, B., Bica, E., Zoccali, M., \& Renzini, A. 2007, A\&A, 470, 1043

Papitto, A., D’Ai, A., Motta, S., Riggio, A., Burderi, L., Di Salvo, T., Belloni, T., \& Iaria, R. 2011, A\&A, 526, L3

Papitto, A., Di Salvo, T., D’Ai, A., Iaria, R., Burderi, L., Riggio, A., Menna, M. T., \& Robba, N. R. 2009, A\&A, 493, L39

Pooley, D., Homan, J., Heinke, C., Linares, M., Altamirano, D., \& Lewin, W. H. G. 2010, ATel, 2974, 1

Proga, D. 2000, ApJ, 538, 684

Ransom, S. M. 2007, in ASP Conf. Ser. 365, Proc. Small Ionized and Neutral Structures in the Diffuse Interstellar Medium, ed. M. Haverkorn \& W. M. Goss (San Francisco, CA: ASP), 265

Revnivtsev, M. G., \& Gilfanov, M. R. 2006, A\&A, 453, 253

Reynolds, M. T., \& Miller, J. M. 2010, ApJ, 723, 1799

Schulz, N. S., Huenemoerder, D. P., Ji, L., Nowak, M., Yao, Y., \& Canizares, C. R. 2009, ApJ, 692, L80

Strohmayer, T. E., \& Markwardt, C. B. 2010, ATel, 2929

Ueda, Y., Murakami, H., Yamaoka, K., Dotani, T., \& Ebisawa, K. 2004, ApJ, 609,325

Verner, D. A., Verner, E. M., \& Ferland, G. J. 1996, At. Data Nucl. Data Tables, 64,1

Wijnands, R., \& van der Klis, M. 1998, Nature, 394, 344 